\title{
EXCITON TRANSPORT IN DISORDERED CRYSTALS: VELOCITY CORRELATION FUNCTIONS ${ }^{1}$
}

\author{
R. PARSON and R. KOPELMAN \\ Department of Chemistry, Unuersity of Michigan, Ann Arbor, Michigan 48109, USA
}

Received 5 March 1984

\begin{abstract}
The two-particle coherent-potential approximation is used to calculate Frenkel-exciton group-velocity correlation functions for substitutionally disordered crystals. Most of the results can be described by a relaxation-time approximation, provided that $k$-dependent and complex relaxation times are allowed; hou ever, some evidence for long-tume tails, associated with the finite frequency range of the scattering potentials, is found. The probable accuracy of the approximation and its relationships w'th localization and kmetic theory are discussed, as is its relevance to experimental systems (triplet excitons in isotopic mixed naphthalene and anthracene crystals)
\end{abstract}

\section{Introduction}

The migration of electronic excitations in substitutionally disordered solids has recently $a^{*}$ tracted much interest [1]. It now appears that most of the experimental observations can be interpreted with randomhopping models [1-4]. Thus is perhaps somewhat puzzling, since the experiments are usually performed at quite low temperatures where there is substantial evidence of delocalized band transport in at least some ordered systems [5]. A plausible explanation for this discrepancy is that in the ordered system hopping and band mechanisms coexist, with the band term dominating at low temperatures, and that disorder suppresses the band component more than it does the hopping one. This suggests that a theoretical estimate of the effect of disorder on pure band transport could provide insight into the problem. In a recent letter [6], we suggested the use of Velicky's two-particle coherent-potential approximation (TCPA) [7] for this purpose; we obtained explicit expressions for the site occupation probabilities and mean-squared displacement and presented a sample calculation of the (long-time) exciton diffusion constant. In the present work, we use this approach to calculate time-dependent transport properties. These are important since they determine the time scales on which the diffusion approximation is valid, and provide information about the mechanism of relaxation into the diffusive regime. We fix our attention upon the configuration-averaged velocity autocorrelation function, a quantity which is easily calculated at this level of approximation and which allows us to make contact with the extensive analytical, numerical, and intuitive experience developed by workers in classical statistical dynamics over the past three decades.

Our present purpose is primarily theoretical: we discuss very detailed dynamic effects which would be difficult or impossible to measure with present experimental techniques, while neglectung phenomena such as phonon scattering which are crucially important in the actual systems. Nevertheless, we believe that our results have experimental significance in that they allow one to quantify statements about the roles of band and hopping transport such as that given above. In the last section of this paper we describe an ad-hoc scheme for combining these results with pure-crystal exciton-phonon data to yield a more realistic and useful description.

1 Supported by NIH Grant No. 2 R01 NS80116-16.

0301-0104/84/\$03.00 (c) Elsevier Science Publishers B.V.

(North-Holland Physics Publishing Division) 


\section{Calculation of correlation functions}

We study the dynamics arising from a tight-binding hamiltonian having site-diagonal disorder:

$$
H=H^{0}+V=\sum_{n}|n\rangle \epsilon_{0}\left\langle n\left|+\sum_{n \cdot n^{\prime}}\right| n\right\rangle J_{n-n^{\prime}}\left\langle n^{\prime}\left|+\sum_{n}\right| n\right\rangle V_{n}\langle n|
$$

in which $|n\rangle$ is a localized site state, $\epsilon_{0}$ is the local-site energy in a perfect crystal, and $J_{n-n^{-}}$is the exciton transfer matrix element connecting sites $n$ and $n^{\prime}$; vector symbols on site and wavevector indices will be omitted. The site potentials $V_{n}$ are independent random variables having zero mean (this can be guaranteed by appropriate choice of $\epsilon_{0}$ ); we will consider specifically a binary random alloy in which they take on values $V_{\mathrm{A}}$ and $V_{\mathrm{B}}$ with respective probabilities $c_{\mathrm{A}}$ and $c_{\mathrm{B}}$.

The conditional probability that an exciton occupies site $n$ at time $t$ given that it occupied site $n^{\prime}$ at time zero is

$$
P_{n n^{\prime}}(t)=\left\langle n\left|\mathrm{e}^{-1 x H}\right| n^{\prime}\right\rangle\left\langle n^{\prime} e^{x t H} \mid n\right\rangle
$$

The Laplace transform of $P(t) . P(u)$, may then be expressed in terms of the one-particle Green function:

$$
\boldsymbol{G}(z)=[z-H]^{-1}
$$

as

$$
P_{n n^{\prime}}(u)=(2 \pi)^{-1} \operatorname{Re}\left(\int \mathrm{d} \omega G_{n \cdot n}(\omega+\mathrm{i} 0) G_{n^{\prime} n}(\omega-\mathrm{i} u)\right)
$$

Since observable properties are related to averages of the probabilities over all configurations of the random potentials, the central task of the theory is to obtain an expression for the configuration average of the product of two Green functions. In ref. [6]. Velicky's two-particle coherent-potential approximation (TCPA) was used for this purpose. This approximation is essentially a self-consistent extrapolation of the standard theory of dilute impurity scattering [8] to higher impurity concentrations. The extension consists of replacing the damped free-particle propagators which appear in the dilute theory by CPA [9] propagators; in order to maintain analyticity and conservation of total probability it is necessary to introduce multiple counting corrections when this is carried out. From the resulting explicit formula for the probabilities an expression for the mean-squared displacement is readily obtained [6]:

$$
\begin{aligned}
& \sum_{n} r_{0 n}^{2} P_{n 0}(u)=N^{-1}\left(n_{c} / u^{2}\right) \sum_{k} v_{k}^{2} \Gamma_{k}^{-1}(u) . \\
& \Gamma_{h}^{-1}(u)=(2 \pi)^{-1} \operatorname{Re}\left(\int \mathrm{d} \omega\left[G_{k}(\omega+\mathrm{i} 0)-G_{k}(\omega-\mathrm{i} u)\right]^{2}\right) .
\end{aligned}
$$

where $n_{c}$ is the lattice coordination number, $v_{h}$ is the group velocity

$$
v_{h}=\nabla_{h} E_{h}=\nabla_{h} \sum_{n} \mathrm{e}^{-i k n} J_{0 n} \text {, }
$$

and

$$
G_{k}(z)=\langle k|G(z)| k\rangle=\left[z-E_{k}-\Sigma(z)\right]^{-1}
$$

is the CPA Green function, which is diagonal in a wavevector basis since it is an approximation to the averaged Green function. The effects of disorder are contained in the self-energy $\Sigma(z)$ which in the CPA is independent of $k$ (local in a site basis). The derivation, properties, and applications of the CPA are discussed at length in standard reviews [9]. 
Although eqs. (2.4) allow an explicit calculation of the mean-squared displacement from the spectral properties of the system, they are rather complicated. The form of (2.4a) strongly suggests that a more natural quantity to study is a group-velocity autocorrelation function (VAF):

$$
\begin{aligned}
& D(t)=N^{-1} \sum_{k}\langle\langle k|v(t) v(0)| k\rangle\rangle_{c}, \\
& \left\langle k|v(t)| k^{\prime}\right\rangle=\sum_{q}\left[\mathrm{e}^{-i t H}\right]_{k q} v_{q}\left[\mathrm{e}^{i s H}\right]_{q k^{\prime}},
\end{aligned}
$$

from which the mean-squared displacement may be obtained by integration. The averaging process in (2.7a) consists of a configuration average, denoted by \langle\rangle$_{c}$, and a "thermal average" which reduces to a trace since the model contains no energy relaxation mechanisms.

By straightforward manipulations we find

$$
D(t)=N^{-1} \sum_{k} v_{k}^{2} C_{k}(t)=N^{-1} \sum_{k} v_{k}^{2} G_{k}^{+}(t) G_{k}^{-}(-t)
$$

in which $G_{k}^{ \pm}(t)$ is the inverse Fourier transform of $G_{k}(\omega \pm i 0)$. We refer to $C_{k}(t)$ as the normalized correlation function (CF); it provides a direct and detailed description of the loss of coherence in an individual $k$ state. Indeed, it is simply a diagonal element of the exciton density matrix in a $k$-basis. (For a careful discussion of the meaning of coherence loss in this context, see ref. [10]. Henceforth, we will use the wavevector $k$ to label the states of the configuration-averaged system; these states have a non-zero energy width or equivalently a finite lifetime. Only in the weak-scattering limit are they simply related to the eigenstates of the pure crystal.) From eq. (2.6) one sees that $C_{k}$ depends on $k$ only through $E_{k}$, so that a single CPA calculation of $\Sigma(z)$ allows one to obtain $C_{k}$ for any $k$. In the language of diagrams one says that there are no vertex corrections to the VAF in the CPA [7].

Some qualitative features of the $\mathrm{CF}$ can be obtained without detailed calculations and factlitate their interpretation. The short-time behavior of $G_{k}(t)$, and thus of $C_{k}(t)$, is determined by the large- $u$ behavior of $G_{k}(\omega+\mathrm{i} u)$, which in turn may be found from the moments of $\Sigma(\omega+\mathrm{i} u)[11]$. We find

$$
C_{k}(t)=1-c_{\mathrm{A}} c_{\mathrm{B}} \Delta^{2} t^{2}+\frac{1}{12} c_{\mathrm{A}} c_{\mathrm{B}} \Delta^{2}\left(\Delta^{2}+2\left(c_{\mathrm{A}}-c_{\mathrm{B}}\right) \Delta E_{k}+\sum_{n} J_{n 0}^{2}-E_{h}^{2}\right) t^{4}+\mathcal{O}\left(t^{6}\right),
$$

where $\Delta=V_{\mathrm{A}}-V_{\mathrm{B}}$. The CF thus has zero slope as $t \rightarrow 0$, as required by dynamical considerations [12]. From an explicit calculation of the first four terms of the short-time expansion of the exact CF:

$$
C_{k}^{\text {exact }}(t)=v_{k}^{-2}\langle\langle k|v(t) v(0)| k\rangle\rangle_{c},
$$

we find agreement to this order. This result is formally pleasant, but of little practical use since the expansion is valid only for times much smaller than the characteristic time for site-to-site transfer. For long times, one can deduce that the CF decays more slowly than an exponential: because of the Herglotz analyticity of $G_{k}(z),\left|G_{k}(t)\right|$ can be obtained from the imaginary part of $G_{k}(\omega)$, which is identically zero outside of a finite range of the $\omega$ axis since the mixed crystal cannot have states in regions where none of its components has any. The Paley-Wiener theorem [13] then implies that $G_{k}(t)$, and hence $C_{k}(t)$, must decay more slowly than an exponential as $t \rightarrow \infty$. (This argument obviously fails for systems where the distribution of $V_{n}$ has tails extending over all energies; an example of this sort is described in the appendix.) The precise form of the long-time tail is determined by the way in which $\operatorname{Im} G_{k}(\omega)$ drops to zero at its band edges. In general this is difficult to determine, but one may set bounds by using the weak-scattering (small $\Delta$ ) limit of $\Sigma(\omega)[10]$ :

$$
\Sigma(\omega)=c_{\mathrm{A}} c_{\mathrm{B}} \Delta^{2} g^{0}(\omega)
$$


where $g^{0}$ is the pure-crystal diagonal Green function. For cubic lattices we find near the edge $\omega_{0}$ :

$$
\begin{aligned}
\operatorname{Im} G_{k}(\omega) & \propto\left(\omega-\omega_{0}\right)^{1 / 2}, & & \text { one dimension; } \\
& \propto\left[\ln \left(\omega-\omega_{0}\right)\right]^{-2}, & & \text { two dimensions; } \\
& \propto\left(\omega-\omega_{0}\right)^{1 / 2}, & & \text { three dimensions. }
\end{aligned}
$$

giving

$$
\begin{aligned}
C_{h}(t) \propto t^{-3}, & \text { one and three dimensions; } \\
\propto t^{-2} . & \text { two dimenisions. }
\end{aligned}
$$

The surprising result that the decay is faster in $1 \mathrm{D}$ than in $2 \mathrm{D}$ can be explained by the divergence of the 1D pure-crystal density of states at the edge. which leads to an enhanced damping of excitations in this region. We may reasonably regard $(2.13)$ as a lower bound on the decay rate of $C_{k}(t)$ in a concentrated mixed crystal; the Paley-Wiener theorem provides an exponential decay as an upper bound. Clearly. these long-time tails are always integrable so that the diffusion constant exists for all non-zero impurity concentrations. Also, the coefficient of the tail is positive, so that it signifies a persistence of dynamical memory through many impurity scattering events. This formal result has little practical significance for transport since the truncation of the spectrum will not strongly affect the shape of $G_{k}(\omega)$ unless $E_{k}$ is near a band edge, where the group velocity is small; it may, however, be important for other time-dependent phenomena, such as dephasing of an optically created wavepacket (this case was discussed in ref. [10] from a somewhat less general standpoint). In this context, we make the trivial, but important, observation that $C_{h}(t)$ as obtained from $(2.8)$ is always positive so that the diffusion constant cannot vanish. The correlated back-scattering processes which lead to a negative-going VAF are not included at this level of approximation.

\section{Results}

We have carried out calculations for a two-dimensional nearest-neighbor square lattice for $\Delta=$ 0.5 (amalgamated bands) and 2.0 (separated bands), and for majority component concentrations $c_{\mathrm{A}}=0.97,0.85$, and 0.60 . (Here and throughout this paper energies and times are measured in units of the pure-crystal half-bandwidth. and lengths in nearest-neighbor lattice spacings.) The CPA equation for a binary alloy is

$\Sigma(z)=\frac{c_{A} c_{B} \Delta^{2} g(z)}{1+\left[\left(c_{A}-c_{B}\right) \Delta+\Sigma(z)\right] g(z)}$.

where

$g(z)=g^{0}(z-\Sigma(z))$,

is the site-diagonal CPA Green function. We used Newton's method to solve (3.1) at 128 or 256 values of $\omega$ evenly spaced within the spectral bounds given by the Saxon-Hutner theorem [9].
As an initial guess we used either the average $t$-matrix approximation [9] or, once the calculation was underway, the result for a neighboring value of $\omega$. This calculation turned out to be quite time-consuming $(\approx 1 \mathrm{~min}$ of CPU time on the Amdahl 5860 at the University of Michigan was required to obtain 128 points) because of the many evaluations of $g^{0}$ involved; while this function is an easily computed elliptic integral for real $z$, its continuation to complex $z$ is more costly to evaluate. (Particular care is needed near the band edges where $\operatorname{Im} \Sigma(z)$ is small but non-zero.) For the $c=0.97$ sample, the number of points calculated was too small to obtain the long-time behavior; to avoid excessive cost we calculated extra points in the vicinity of the band edges and then interpolated within the band, where $\Sigma(\omega)$ varies slowly, to get a total of 2048 points. Once $\Sigma$ was obtained, $C_{h}(t)=C\left(E_{k}, t\right)$ was calculated for 80 values of $E_{k}$ using a fast Fourier transform and $D(t)$ obtained from eq. (2.4). To simplify the wave-vector integration, we first calculated a 
velocity-weighted density of states function:

$\rho_{v}(\nu)=N^{-1} \sum_{k} \delta\left(\nu-E_{k}\right) v_{k}^{2}$,

$D(t)=\int \mathrm{d} \nu \rho_{v}(\nu) C(\nu, t)$

By using the Fourier representation of the $\delta$-function, $\rho_{v}(\nu)$ can be reduced to integrals over products of Bessel functions which may in turn be related to off-diagonal lattice Green functions. In two dimensions the latter can be expressed in terms of the complete elliptic integrals $K$ and $E$ [14], giving finally:

$$
\begin{aligned}
\rho_{v}(\nu)= & \left(4 / \pi^{2}\right)\left[E\left(\left(1-\nu^{2}\right)^{1 / 2}\right)\right. \\
& \left.-\nu^{2} K\left(\left(1-\nu^{2}\right)^{1 / 2}\right)\right] .
\end{aligned}
$$

This function decreases smoothly with negative curvature from $4 / \pi^{2}$ at $\nu=0$ to 0 at $\nu= \pm 1$.

Figs. 1 and 2 show typical results for $C_{k}(t)$. In the separated-band case (fig. 1b), a high-frequency oscillation appears; this clearly arises from the fact that for each value of $E_{k}$ the spectral density has a component in each subband, so that $C_{k}$ is a coherent superposition of contributions from these components. Except for this, most of the curves are well represented by exponentials over most of the time domain for which they were calculated. The quadratic short-time asymptote is hard to see in the figures but can be verified by a close examination of the results. The long-time tails, however, are very hard to see except in the nearly pure crystal; in the concentrated crystals, the decay of $C_{k}$ is approximately exponential over 8-12 orders of magnitude, after which it is lost in numerical noise. In general, we found that the decay of $C_{k}(t)$ is fastest near the center of the band and slowest near the edges. In the amalgamated-band case, it decreases smoothly from the center towards the edges, as one expects from perturbation theory, while in the separated-band case the variation is rather irregular, again reflecting the superposition of two components for each $k$.

Fig. 3 shows our results for the VAF $D(t)$ obtained from eq. (3.3). One can see that the oscillatory structure is not entirely washed out by averaging over $k$. This is expected since if the
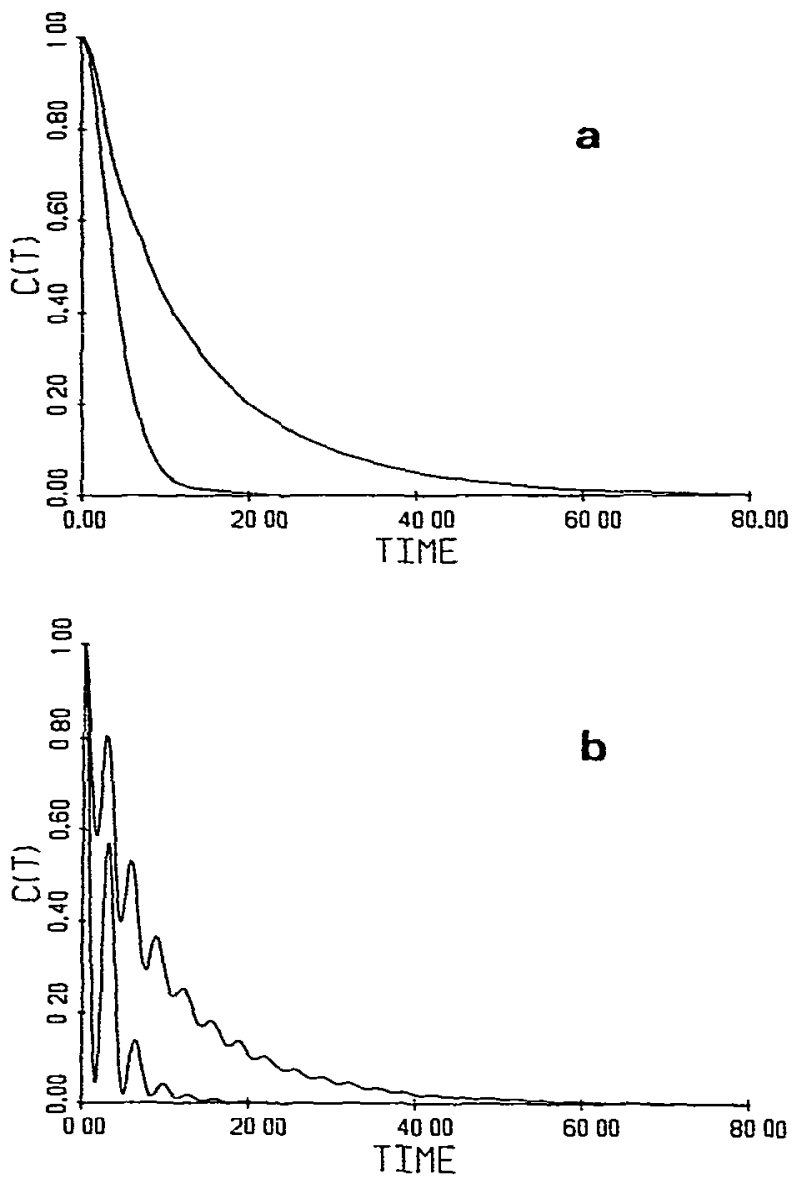

Fig. 1. Normalized correlation function $C_{k}(t)$ at $E_{k}=0$ for $c=0.60$ (left curve) and $c=0.85$ (right curve), and (a) $\Delta=05$, (b) $\Delta=2.0$.

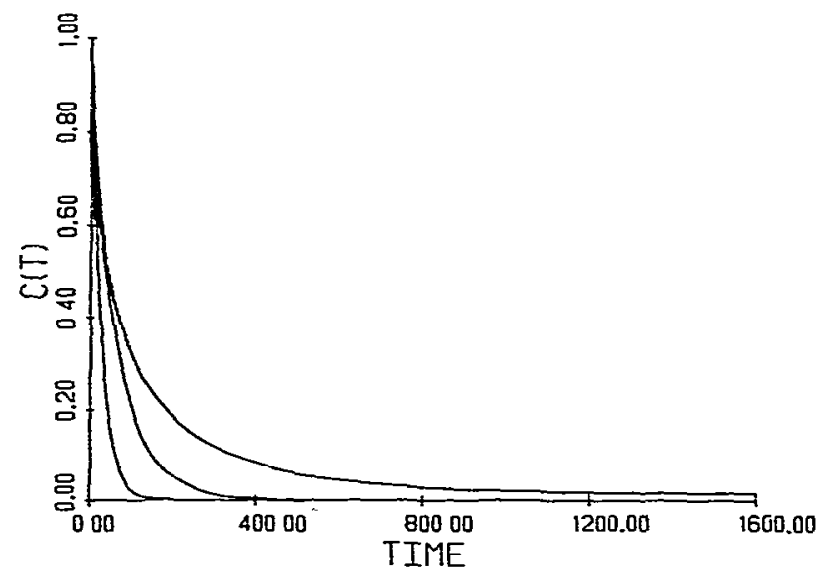

Fig. 2. Normalized correlation function $C_{k}(t)$ for $c=0.97$, $\Delta=2$, at (lefi to right) $E_{k}=0,0.5$ and 1 . 

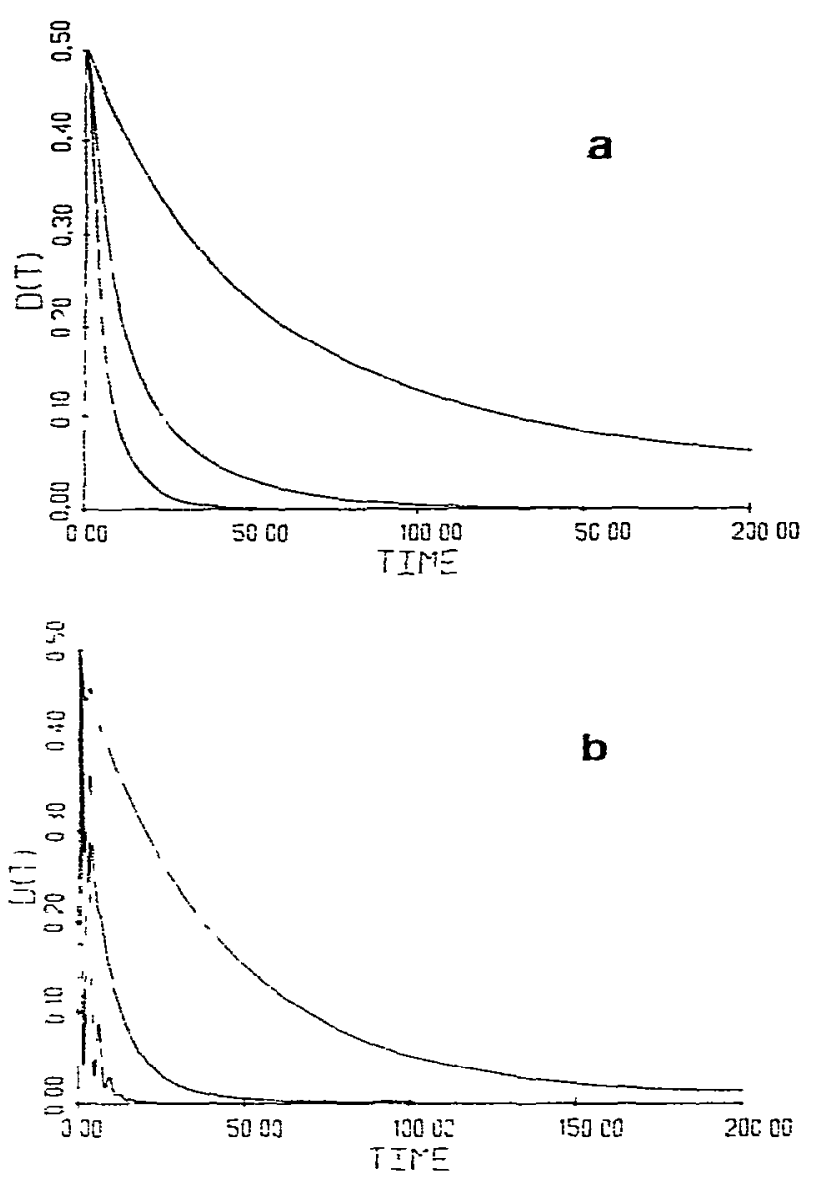

Fig. 3. Velocity correlation function $D(t)$ for $(a)\lrcorner=05$. (b) $1=20$. and (left to $\mathrm{nght}$ ) $c=060.0 \mathrm{S5}$ and 097 .

bands are well separated the separation between spectral density components is roughly constant $(\approx \Delta)$ over the band. so that the oscillations add up coherently (the structure was unchanged when the number of points used in the integration was increased by a factor of four. so that we are confident that it is not a discretization artifact).

In table 1 we show our final results for the diffusion constant $D$; these were obtained from eq. (2.4) evaluated at $u=0$ rather than from $D(t)$ (to avoid an unnecessary Fourier transform). In light of what has been discussed, these results contain no surprises: the diffusion constant increases rapidly as the impurity concentration becomes small, and decreases with increasing $\Delta$ due to reduced tunneling through impurities.
Table 1

Diffusion constants

\begin{tabular}{llrl}
\hline 1 & \multicolumn{2}{l}{ Concentration } & \\
\cline { 2 - 4 } & 0.97 & 0.85 & 0.60 \\
\hline 0.5 & 94.6 & 15.5 & 66 \\
2.0 & 442 & 66 & 24 \\
\hline
\end{tabular}

\section{Discussion}

We address here two distinct points: the extent to which our calculations correctly describe the dynamics of the model (2.1). and the extent to which they can be used to interpret the behavior of experimental systems. A comprehensive study of the first point would involve comparison with exact numerical results, which are not now available. Such calculations would be very interesting. though rather difficult to carry out because the quantities of interest are non-local in space. The mean-squared displacement would be straightforward to calculate by the method which Prelovsek [15] has used for the Anderson model, but it would be less informative than the VAF itself, and it would be difficult in practice to extract the VAF from the mean-squared displacement unless a very short time step were used in the numerical calculation. In the absence of exact calculations, we can compare our results to those limiting cases which are reasonably well understood. In the weakscattering and low-density limits, the TCPA reduces to the well-known perturbative expressions [7]. For sufficiently strong disorder, we know that the exact eigenstates will become exponentially localized, and the diffusion constant must vanish; as mentioned in section 2 this cannot occur in this approximation, although in practice the diffusion constant can become extremely small. The problem of localization in a binary alloy is quite complicated and no precise estimates, either from theory or from numerical calculations, of the fraction of localized states are available except in the limit $\Delta \rightarrow \infty$ (quantum percolation [16]). It might appear that our approach will always fail for large enough $\Delta$, regardless of concentration, since at least one of the subbands will contain many localized states. However, at low impurity concentra- 
tions the contribution of the impurity (minority component) subband to the diffusion constant is negligible so that this defect is unimportant. We expect then that in the large- $\Delta$ limit our results will be accurate away from the quantum percolation edge, which very nearly coincides with the classical edge [16]; as $\Delta$ decreases the range of concentrations for which this approximation is useful will increase, and when the subbands are merged $(\Delta=$ 1) we will have an accurate description for all concentrations.

The discussion above implicitly assumed a spatial dimensionality greater than two, since perturbation theory, of which the TCPA is an extrapolation, breaks down for $d \leqslant 2$ [17]. In one-dimensional disordered systems, all states are exponentially localized and this theory will be useless for them. Although it is now generally accepted that extended states do not exist in two-dimensional disordered systems (in the presence of time-reversal symmetry) [18], the length and time scales associated with this peculiar phenomenon are exiremely large. The absence of quantum diffusion in two dimensions arises from a small, negative $1 / t$ component in the VAF $[17,19]$ which would probably be invisible on our time scales since it is very difficult to detect in numerical calculations on the Anderson model [15]. (Of course, it would eventually wipe out the positive long-time tails we have discussed; the competition between these tails would make a definite numerical identification of either a very difficult task.) In the experimental systems, either inelastic scattering by phonons or the finite exciton lifetime will cut off the long-time anomalies; the overall result will be that our diffusion constants will be somewhat too large at all concentrations in two-dimensional systems.

In the dilute impurity limit, the two-dimensional anomalies arise from a class of terms, the "maximally subcrossed diagrams" [17], which are not included in the TCPA. This suggests that a more powerful theory could be obtained by incorporating CPA propagators into these diagrams in the same way that they were inserted into the dilute limit ladder diagrams to give the TCPA. Unfortunately, this procedure leads to expressions which fail to conserve total probability, because the one- and two-particle propagators are not mutually consistent; additional scattering events are accounted for in one but not in the other. The formal expression of this consistency condition is a "Ward-identity" relating the two propagators, which is satisfied in the TCPA but violated by the new terms, even in the dilute limit [20]. Chitanvis and Leath [20] have presented a systematic scheme for incorporating multiple scattering into the TCPA which obeys the "Ward identities" at every level of approximation; it is not clear, however, whether the new terms include those which one expects to be important for transport. A numerical study of the lowest-order Chitanvis-Leath approximation would be interesting, though rather involved.

Having discussed the defects of the TCPA at some length, we emphasize here its positive features: it incorporates a convenient and accurate description of the spectral properties of a random alloy, the CPA, into a transport theory in a selfconsistent way. The calculations are carried out upon a discrete lattice, for which excluded-volume effects are completely accounted for by the multiple counting corrections, and the exact single-site $t$-matrix is used so that the penetrable nature of the scatterers for $\Delta<\infty$ is included. The overall scheme is analogous to the Boltzmann-Enskog kinetic theory of dense gasses: genuine many-body correlations are neglected, but some of their more obvious (excluded-volume) physical consequences are grafted onto a low-density theory [21].

Turning now to experimental systems, we first remark that the hamiltonian (2.1) is grossly incomplete as it contains no mechanism for energy relaxation. For excitons, energy relaxation is provided by the exciton-phonon interaction (which also, of course, provides an additional phase relaxation channel). We have recently carried out a detailed theoretical study of exciton-phonon interactions in disordered systems [22]; the results, however, are either quite formal or require tedious calculations involving many unknown parameters if they are to be applied realistically. We suggest here an ad-hoc procedure, which can to some extent be justified by the formal theory, for building known exciton-phonon information into a rigid-lattice theory: multiply each $C_{k}(t)$ by 
$\exp \left(-2 \gamma_{k} t\right)$, where $\gamma_{k}$ is the total rate of scattering out of state $|k\rangle$ in the pure crystal, which at low temperatures can be calculated in a one- or twophonon approximation. We must then modify the definition of the VAF so that it mimics a canonical distribution over the exact eigenstates of the disordered system. This step is in general ill-defined: it is obviously wrong simply to introduce a factor $\exp \left(-\beta E_{k}\right)$. except in the weak-scattering limit, since in a strongly disordered system $E_{k}$ is not an energy. If the bands are well separated, however, there will be a range of temperatures for which the equilibrium population of the upper subband is negligible. One can then discard the upper-subband contributions to the transport entirely, and weight the lower-subband contributions by $\exp \left[-\beta\left(E_{k}+\bar{\Sigma}^{r}\right)\right]$, where $\bar{\Sigma}^{r}$ is the average value of the real part of the self-energy in the lower subband. (In the split-band limit $\Sigma^{\mathrm{r}}(\omega)$ varies very little within a subband; its principal function is to shift the entire subband away from the virtual crystal energy at which $E_{k}$ is defined.) Clearly, this procedure will only work if the majortty subband is lower in energy: in the opposite case, the minority subband, which the TCPA treats poorly, will be entirely responsible for the transport.

To illustrate these ideas, we have calculated the self-energy for the well-studied first triplet exciton of $\mathrm{C}_{10} \mathrm{H}_{8} / \mathrm{C}_{10} \mathrm{D}_{8}$, using a square-lattice approximation to the actual band structure. This case is especially simple since the guest-host separation is so large $\left(93 \mathrm{~cm}^{-1}\right.$ for a bandwidth of $10 \mathrm{~cm}^{-1}$ [23]. or 18.6 in our units) that the imaginary part of the self-energy varies very slowly over the lower subband except very near the edges which are irrelevant for transport. We can then define a single disorder scattering time for the subband: velocity correlations will decay exponentially with a rate $-2 \operatorname{Im}(\Sigma)$. Moreover, we can use the much simpler average $t$-matrix approximation (ATA) [9] instead of the CPA since the shape of the subband is not important. In fig. 4 we show the average ATA self-energy for the lower subband in the low impurity concentration regime. The value of $\Sigma$ at $c=0.15$ yields a scattering time on the order of four intersite transfers, so that at this concentration disorder has essentially extinguished coherent

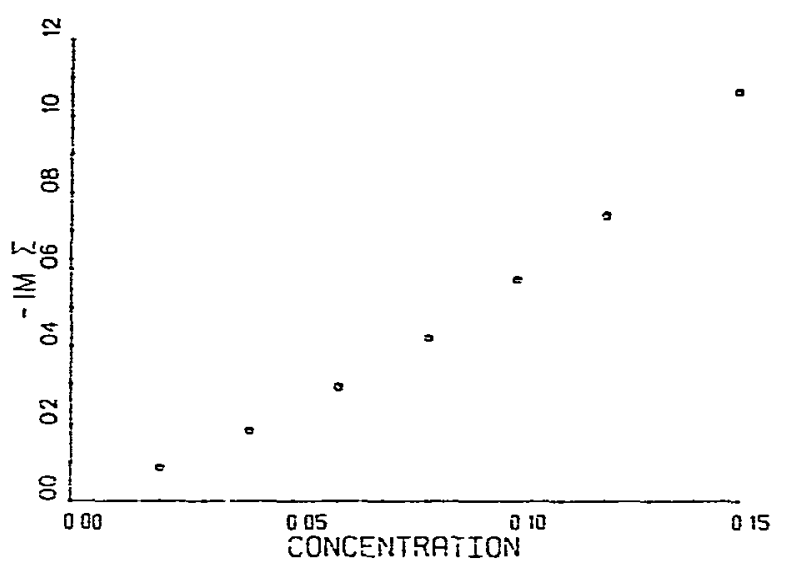

Fig 4. Imagindry self-energy (averaged over the lower subband) for the first inplet exciton of a $\mathrm{C}_{10} \mathrm{H}_{8} / \mathrm{C}_{10} \mathrm{D}_{8}$ mixed crystal. for several impurity $\left(C_{10} D_{B}\right)$ concentrations For this system. one energy unit is $=5 \mathrm{~cm}^{-1}$. A y-axis value of 0.01 then corresponds to an interval of $\approx 50$ intersite transfer tumes betueen scattering events.

effects. By comparing these results to the phonon scattering rate, estimated from the optical linewidth, of $\approx 0.1 \mathrm{~cm}^{-1}$ at $7 \mathrm{~K}(0.02$ in our units) [24], one can estimate that phonon scattering will prevail for impurity concentrations less than $=$ 0.05 . Since the optical lineshape only measures the $k=0$ scattering, and since one expects on general grounds (increased density of states) a somewhat larger rate within the band, the crossover to behavior dominated by disorder scattering might be closer to $10 \%$. In contrast, at $1.8 \mathrm{~K}$ the optical linewidth of the first triplet state of anthracene, which has a bandwidth of $20.5 \mathrm{~cm}^{-1}$, is $\approx 0.009$ $\mathrm{cm}^{-1}$ [25], or 0.0008 in our units. A perdeuteroanthracene concentration of only 0.002 yields a self-energy of about this size (again using the ATA, which is certainly valid in this concentration regime), so that at this temperature disorder scattering dominates except in an extremely pure crystal. The available data concerning excitonphonon scattering in two- or three-dimensional crystals at low temperatures are scanty and their interpretation subject to considerable uncertainty, so that more precise statements are unwarranted at this time.

In conclusion, we believe that the approach presented and illustrated here allows one to make a reasonable estimate of the extent to which static disorder alone can destroy transport coherence. 
When one observes the amount by which the transport in a $85 \%$ crystal has been reduced, it comes as no surprise that in a real system with phonon scattering coherence effects are negligible, as is suggested by the available experimental data on transport in mixed crystals [26]. In the course of this investigation, we have produced a good deal of very detailed dynamic information which may be of use for comparison to numerical calculation or, eventually, to experiment.

\section{Appendix: Results for the Lloyd model}

Lloyd [27] has shown that the exact configuration-averaged one-particle Green function can be found for the hamiltonian (2.1) if the probability density of the random site potential $V_{n}$ takes the Cauchy (Lorentz) form

$P\left(V_{n}\right)=\sigma / \pi\left(\sigma^{2}+V^{2}\right)$.

For this case, the exact self-energy, which coincides with the CPA self-energy, is amazingly simpie

$\Sigma(\omega \pm \mathrm{i} u)=\mp \mathrm{i} \sigma$.

From the TCPA, which is still an approximation even though the CPA is exact, one readily finds

$D(t)=C_{k}(t) / 2=\frac{1}{2} \mathrm{e}^{-2 \sigma t}, \quad D=1 / 4 \sigma$.

The pure exponential autocorrelation implies that the exciton velocity, interpreted as a random process, is markovian on all time scales, like the velocity of a brownian particle in the Langevin approximation [12]. The mean-squared displacement initially varies at $t^{2}$, crossing over to diffusive behavior on the time scale $1 / 2 \sigma$.

Because of the long tails of the Cauchy distribution, the result (A.3) conflicts with the short- and long-time considerations of section 2 . The moment expansion which gave the short-time limit fails since the moments are infinite. Indeed, the infinite variance of the distribution implies that the model has no genuine weak-disorder limit; for arbitrarily small $\sigma$ there are many sites having large $V_{n}$. Moreover, the spectral density develops an $\omega^{-2}$ tail which leads to pure exponential decay in its transform; in effect, every impurity collision involves a complete loss of memory. In contrast, in the binary alloy there is a persistence of dynamical behavior, shown by the long-time tails, although in practice this effect is small.

\section{References}

[1] R. Kopelman, in: Spectroscopy and excitation dynamics of condensed molccular systems, eds V.M. Agranovich and R.M. Hochstrasser (North-Holland, Amsterdam, 1983).

[2] C.R. Gochanour, H C. Andersen and M.D. Fayer, J. Chem. Phys 70 (1979) 4254;

R F. Loring, H.C Andersen and M D. Fayer, J. Chem. Phys. 76 (1982) 2015; J. Chem. Phys 80 (1984) 5731, Phys. Rev Letters 50 (1983) 1324.

[3] T. Odagakı and M. Lax, Phys. Rev. B24 (1981) 5284;

1. Webman, Phys. Rev. Letters 47 (1981) 1496;

G. Korzentewski, R. Friesner and R. Silbey, J. Stat. Phys 31 (1983) 451;

M. Sahimi, B.D. Hughes, L.E. Scriven and H.T. Davis, J. Chem Phys 78 (1983) 6489.

[4] B Movaghar and W. Schirmacher, J. Phys. C14 (1981) 859;

B Movaghar, M. Grünewald, B. Pohlmann, D. Würtz and W. Schirmacher, J. Stat. Phys. 30 (1983) 315.

[5] M D. Fayer, in- Spectroscopy and excitation dynamics of condensed molecular systems, eds. V.M. Agranovich and R.M. Hochstrasser (North-Holland, Amsterdarn, 1983);

A. Zewal, in: Excitons, eds. E. Rashba and M.D. Sturge (North-Holland, Amsterdam, 1982);

A J. van Strien, J. Schmidt and R. Silbey, Mol. Phys. 46 (1982) 151.

[6] R. Parson, Chem. Phys. Letters 100 (1983) 57.

[7] B. Velicky, Phys. Rev. 184 (1969) 614; P. Leath, Phys. Rev. B2 (1970) 3078.

[8] S Doniach and EH Sondhermer, Green's functions for solid-state physicists (Benjamin, Reading, 1974).

[9] R. Elliot, J. Krumhansl and P. Leath, Rev. Mod. Phys. 46 (1974) 465 .

[10] I. Abram and R.M. Hochstrasser, J Chem. Phys. 72 (1980) 3617.

[11] B. Velicky, S. Kirkpatrick and H. Ehrenreich, Phys. Rev. 175 (1968) 742;

H K. Hong and R. Kopelman, J. Chem Phys. 55 (1971) 3491.

[12] S.W. Lovesey, Condensed matter physics. dynamic correlations (Benjamm, Reading, 1980).

[13] R E.A.C. Paley and N. Wiener, Fourier transforms in the complex domain (Am Mat. Soc., New York, 1934) p. 24.

[14] T. Monta, J. Math. Phys. 12 (1971) 1744

[15] P. Prelovsek, Phys. Rev B18 (1978) 3657.

[16] T. Odagaki, N. Ogita and H Matsuda, J Phys C13 (1980) 189; 
S. Kirkpatrick, in-Ill-condensed matter, eds. R. Balian, R. Maynard and G. Toulouse North-Holland. Amsterdam. 1979).

[17] E Abrahams, P.W. Anderson and T. Ramakrishnan, Phil. Mag B42 (1980) 827;

L.P. Gorkov, D. Khmelnitskii and A. Larkin, JETP Letters 30 (1979) 228.

[18] E. Abrahams. P.W. Anderson, DC. Licciardello and T. Ramakrishnan. Phys. Rev. Letters 42 (1979) 673.

[19] T. Kirkpatrick and J.R. Dorfman. Phys. Rev. A28 (1983) 1022.

[20] S. Chitanvis and P Leath. J. Phys. C15 (1982) 3513; 16 (1983) 1049.
[21] J.R. Dorfman, in: Studies in statistical mechanics, Vol. 9, ed. H.J. Raveche (North-Holland, Amsterdam, 1981)

[22] R. Parson, J. Chem. Phys. 81 (1984), to be published.

[23] EM. Monberg and R. Kopelman, Mol. Cryst. Liquid Cryst. 57 (1980) 271.

[24] H. Port and D. Rund, J. Mol. Struct. 45 (1978) 455.

[25] H. Port, in: Organic molecular aggregates, eds. P. Reineker, H. Haken and H.C. Wolf (Springer, Berlin, 1983).

[26] S. Gentry and R. Kopelman, J. Chem. Phys. 78 (1983) 373; Phys. Rev. B27 (1983) 2579; to be published.

[27] P. Lloyd. J. Phys. C2 (1969) 1717. 